\title{
Qualidade de vida dos cuidadores de amputados de membros inferiores
}

\author{
Quality of life of lower limb amputees' caregivers
}

\section{Marcos Henrique Dall'Aglio Foss ${ }^{1}$, Marielza R Ismael Martins ${ }^{2}$, Lisabelle Manente Mazaro ${ }^{3}$, Mariana I Dias Martins'4, José Maria Pereira Godoy 5}

\section{RESUMO}

Introdução. A amputação de membros inferiores ainda é hoje um dos maiores problemas de saúde pública no Brasil. O longo período do processo de reabilitação faz com que a dependência desses pacientes exija quase que na totalidade de cuidadores. Objetivo. Avaliar a sobrecarga nos cuidadores de pacientes submetidos à amputação. Método. Avaliou-se em estudo prospectivo e aleatório 87 cuidadores de pacientes amputados no ambulatório de cirurgia vascular de um Hospital escola. Utilizou-se o Caregiver Burden Scale (CBE) para avaliação da sobrecarga do cuidador e uma entrevista semiestruturada para caracterização deste e do paciente. Resultados. Os cuidadores eram, em sua maioria, mulheres (83,3\%), casadas $(65,2 \%)$, média de idade de 40,5 anos, na condição de esposas $(54,6 \%)$ e mães $(32,1 \%)$ e moderadamente sobrecarregadas ( $C B E$ total $=2,33$ ). As variáveis tensão geral, isolamento e envolvimento emocional estavam abaixo da média em 41 cuidadores (47,5\%) e decepção e ambiente, em 39 pacientes (45\%). As variáveis tensão geral e isolamento versus emoção e emoção versus ambiente mostraram associação $(p<0,05)$. Conclusão. Este estudo fornece subsídios à equipe multiprofissional mostrando que são necessárias políticas públicas efetivas, destinadas a oferecer uma rede de serviços de suporte para que melhore a qualidade de vida de todos.

Unitermos. Cuidadores, qualidade de vida, Amputação

Citação. Foss MHDA, Martins MRI, Mazaro LM, Martins MID, Godoy JMP. Qualidade de vida dos cuidadores de amputados de membros inferiores.

Trabalho realizado no Ambulatório de Cirurgia Vascular do Hospital de Base de São José do Rio Preto - Faculdade de Medicina de Rio Preto - FAMERP

1. Fisioterapeuta, Departamento de Ciências Neurológicas da Faculdade de Medicina de Rio Preto - FAMERP, São José do Rio Preto-SP, Brasil.

2. Terapeuta Ocupacional, Doutora, Departamento de Ciências Neurológicas da Faculdade de Medicina de Rio Preto - FAMERP, São José do Rio Preto-SP, Brasil.

3. Terapeuta Ocupacional, Coordenadora do Curso de Terapia Ocupacional da Fundação Educacional de Fernandópolis - FEF, Fernandópolis-SP, Brasil.

4. Acadêmica de Medicina da Universidade do Vale do Sapucaí UNIVAS, Pouso Alegre-MG, Brasil.

\section{SUMMARY}

Introduction. The amputation of lower limbs is still one of the major public health problems in Brazil. The process of rehabilitation means that the dependence of these patients is increasing, requiring almost all of the caregivers. Objective. To evaluate the quality of life of family caregivers of patients with amputation. Method. It is a prospective study and random 87 Caregivers of lower limb amputees of vascular surgery at the clinic of an School Hospital. We used the Caregiver Burden Scale (CBE) to evaluate the quality of the caregiver burden and a semi-structured for characterization of the family caregiver and the patient. Results. The caregivers were, in their majority, women $(83.3 \%)$, married (65.2\%), with mean age of 40.5 years. As a spouse (54.6\%) and mothers (32.1\%) and were moderately burdened (CBE total $=2.33$ ). The variables general tension, isolation and emotional involvement were below the median in 41 caregivers (47.5\%) and disappointment and environment in 39 patients (45\%). General tension and isolation versus emotion and emotion versus environment showed association $(p<0.05)$. Conclusion. This study provides subsidies will team showing that effective public policies are necessary, to provide a network of support services for improving the quality of life for all.

Keywords. Caregivers, Quality of Life, Amputation. Citation. Foss MHDA, Martins MRI, Mazaro LM, Martins MID, Godoy JMP. Quality of life of lower limb amputees' caregivers.

Endereço para correspondência Departamento de Ciências Neurológicas Av. Brigadeiro Faria Lima, 5416 CEP 15090-000, São José do Rio Preto-SP, Brasil. E-mail: marielzamartins@famerp.br 


\section{INTRODUÇÃO}

A mais devastadora conseqüência da doença arterial degenerativa dos membros inferiores é a amputação, tornando-se importante problema de saúde pública, no Brasil e no mundo ${ }^{1,2}$.

Observa-se que ainda há muita demora no início do processo de reabilitação; que a média de tempo de tratamento é maior que o descrito na literatura, para quase todos os níveis de amputação; que os índices de abandono de tratamento são elevados, mesmo nas amputações de membros inferiores; que ainda há muita inadequação e desconhecimento, por parte dos médicos, em relação ao processo de reabilitação de pacientes amputados ${ }^{3}$.

Esses pacientes tornam-se dependentes de cuidadores e estes nem sempre estão orientados a exercer a nova função porque são remetidos na maioria das vezes a essa condição de forma inesperada. Define-se como cuidador primário a pessoa que possui a responsabilidade de cuidados com o paciente e ainda divide seu tempo com outras responsabilidades ${ }^{4,5}$. Outras definições afirmam que cuidador formal é aquele que possui responsabilidade diariamente com o paciente e o informal presta ajuda ocasional6. Um estudo avaliando a importância da enfermagem em recorrer à estratégia de ensino-aprendizagem para orientar o familiar/cuidador no processo do cuidar de pessoas em condição crônica e com a finalidade de promover a vida, apesar da doença, mostrou que a qualidade desse cuidado prevenirá recidivas e conseqüentes reinternaçöes ${ }^{7}$.

Avaliando cuidadores de pacientes submetidos á hemodiálise detectou-se que os aspectos emocionais dos cuidadores (particularmente das esposas) e dos pacientes são importantes fatores preditivos de sobrecarga. Intervenção de suporte social e psicológica deve ser considerada a fim de melhorar a qualidade de vida dos cuidadores e dos pacientes a eles vinculados ${ }^{8}$.

Um estudo também se preocupou em investigar a avaliação subjetiva de cuidadores por meio de percepções positivas e negativas com relação ao cuidado 9 . Para isto elaboraram um inventário de ônus e benefícios associados ao cuidado. Constataram que, apesar da sobrecarga de trabalho, problemas de saúde, insônia, cansaço, falta de atividades de lazer, comprometimento da atividade profissional, a percepção da qualidade de vida (QV) foi elevada em aspectos como senso de autorealização e auto-reconhecimento.
Assim, muitos aspectos analisados como insatisfatórios por meio de indicadores objetivos podem ser subjetivamente considerados satisfatórios pelos indivíduos em questão. Tanto a dimensão objetiva quanto a subjetiva é importante para o enriquecimento de informações coletadas com a finalidade de avaliar a QV de cuidadores, permitindo melhor interpretar sua realidade de vida e saúde $^{10}$.

Com esse objetivo, surgiram medidas como o Caregiver Burden Scale (CBE), validado no Brasil e, trata-se de um instrumento utilizado para avaliar a sobrecarga subjetiva dos cuidadores de doenças crônicas, cujos escores variam de 1 a 4, sendo que os valores maiores indicam uma maior sobrecar$\mathrm{ga}^{11}$.

O presente estudo buscou avaliar a QV por meio das percepções subjetivas dos cuidadores com relação às diversas dimensões da vida. $\mathrm{Na}$ entrevista semi-estruturada foram abordados indicadores sociais e de saúde e a sobrecarga percebida pelo cuidador em decorrência do cuidar para investigar sua condição de cuidador de pacientes submetidos à amputação.

\section{MÉTODO}

Amostra

Trata-se de um estudo descritivo, transversal, de abordagem quantitativa.

Foram avaliados os aspectos gerais da qualidade de vida em 87 cuidadores de pacientes amputados de membros inferiores no ambulatório de cirurgia vascular do Hospital de Base de São José do Rio Preto, vinculado á Faculdade de Medicina FAMERP, no período de dezembro de 2007 á março de 2008. Foram realizados levantamentos de cuidadores de pacientes amputados no último ano que retornaram ao tratamento pós-amputação no serviço de angiologia e cirurgia vascular do Hospital de Base. A partir do levantamento os indivíduos foram convidados a comparecerem no ambulatório de Cirurgia Vascular onde foram comunicados sobre a realização da pesquisa e após concordarem e assinarem o Termo de Consentimento, responderam ás questões.

Todos os pacientes estavam em processo de reabilitação, realizando enfaixamento, desensibilização, posicionamento do coto em extensão, alongamento muscular, exercícios de fortalecimento e ADM e treino marcha. Nenhum protetizado. 
Os critérios utilizados na seleção dos sujeitos da pesquisa foram: familiares maiores de 16 anos, prestando cuidados há mais de dois meses à pessoa amputada dependente para as atividades de vida diária. Foram excluídas famílias onde não se identificou o cuidador primário. $O$ estudo obteve aprovação da Comisão de Ética em Pesquisa da FAMERP (228/07).

\section{Procedimento}

Para avaliação do impacto nos cuidadores dos amputados foi utilizado o Caregiver Burden Scale (CBE) ${ }^{11}$, validado no Brasil em 1998, que avalia cinco itens (tensão geral, isolamento, decepção, emocional, ambiente) e tem escores 1 para menor efeito e 4 para grande efeito. Também foi realizada entrevista para caracterizar dados sociodemográficos.

O tempo necessário para aplicação do instrumento (CBE) e para a entrevista foi de 45 minutos aplicados por entrevistadores treinados

\section{Análise Estatística}

Foi usada análise de variância e média com nível de significância de 0,05, comparando as variáveis relacionadas à sobrecarga do cuidador, com auxílio do pacote estatístico SPSS-PC (Statistical Package for Social Sciences, versão 8.0, Chicago, IL, USA).

\section{RESULTADOS}

No grupo de pacientes com amputação de membros inferiores, 56 eram do sexo masculino (64\%), com média de idade de 32,5 anos, com período de amputação variando entre 4 meses e 1 ano.

No grupo dos cuidadores o papel de cuidador primário foi atribuído a: 28 mães (32,1\%), 6 pais (6,8\%), 40 esposas (54,6\%), 4 irmãs $(4,5 \%), 1$ neta (1\%), 1 noiva (1\%). A idade variou entre 27 a 73 anos e a média de idade foi 40,05 anos, sendo $83 \%$ do sexo feminino e $17 \%$ do sexo masculino. Quanto a escolaridade, $48 \%$ possuem ensino fundamental incompleto (média de 5 anos de estudo).

A sobrecarga na vida dos cuidadores avaliados neste estudo mostrou os seguintes valores médios dos escores ( $1=$ de modo algum e $4=$ frequentemente) respectivamente: 1,99 (tensão geral); 2,165 (isolamento); 2,0 (decepção); 1,33 (emocional); 2,33 (ambiente).
As variáveis tensão geral, isolamento e envolvimento emocional estavam abaixo da média em 41 cuidadores (47,5\%) e decepção e ambiente em 39 pacientes (45\%) (Gráfico 1). Verifica-se que a dimensão de envolvimento emocional mostra-se com menor sobrecarga quando avaliada isoladamente.

As variáveis tensão geral e isolamento versus emoção mostraram associação $(p<0,05)$ e emoção versus ambiente também $(p<0,05 ;$ Tabela 1$)$.

Gráfico 1. Média e desvio padrão das dimensões da Escala CBE encontradas no grupo de cuidadores.

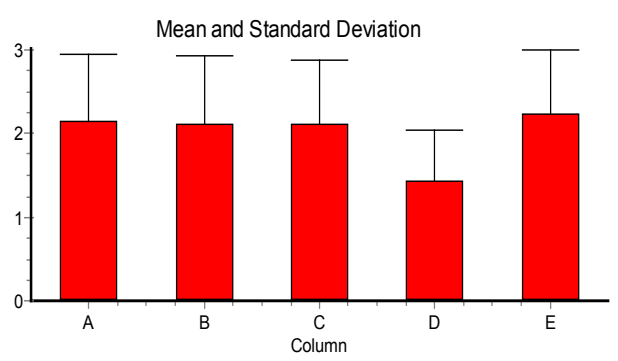

$\mathrm{A}=$ Tensão Geral; $\mathrm{B}=$ Isolamento; $\mathrm{C}=$ Decepção; $\mathrm{D}=$ envolvimento emocional; $\mathrm{E}=$ Ambiente.

\section{DISCUSSÃO}

De maneira geral, o resultado deste estudo mostrou que os sujeitos da casuística, a partir do momento. Este estudo, que investigou a QV dos cuidadores de amputados de membros inferiores atendidos no ambulatório de cirurgia vascular de um hospital-escola, mostrou que cuidadores de amputados sofrem interferência dessa atividade.

Considerou-se a QV de vida como um construto multidimensional, de natureza objetiva e subjetiva, determinado socialmente pelas condições de vida e trabalho dos indivíduos e relativizado pelo contexto histórico e cultural no qual se inserem.

$\mathrm{O}$ instrumento de medida $(\mathrm{CBE})$ associado à entrevista semi-estruturada procuraram identificar dados objetivos, subjetivos e específicos do cuidador e das diversas dimensões da vida, comuns a pacientes e cuidadores.

O impacto negativo na vida dessas pessoas foi detectado nesse estudo. $\mathrm{O}$ aspecto emocional quando correlacionado com os aspectos como tensão geral, isolamento, decepção e ambiente foram significantes. Tal fato sugere que estes cui- 
dadores necessitam de orientação e suporte psicológicos.

A maioria dos cuidadores nesse estudo era do sexo feminino, principalmente esposa e filha. Apenas $17,5 \%$ dos pacientes tinham cuidadores não familiares e remunerados. Desses cuidadores $42,5 \%$ não tinham remuneração do paciente ou outra fonte de renda. Isso sugere que esses cuidadores são dependentes economicamente de outras pessoas podendo, portanto, afetar esses resultados.

Não foram encontrados estudos que permitissem comparar as características de pacientes amputados, pois os trabalhos encontrados na literatura são específicos para pacientes idosos ${ }^{12,13}$.

Outro aspecto importante é que $15 \%$ desses cuidadores e $42,5 \%$ dos pacientes são analfabetos, sugerindo maior dificuldade em relação aos cuidados. Em relação a este resultado a escolaridade é predominantemente baixa em vários estudos com cuidadores em âmbito nacional ${ }^{14,15}$ e internacional ${ }^{16,17}$. O baixo nível de escolaridade pode contribuir para que estas pessoas assumam o papel de cuidador visto que a inserção no trabalho formal para pessoas com baixa escolaridade é mais difícil.

Houve relato de problema de saúde em $22,5 \%$ dos cuidadores, mostrando uma situação contraproducente e que também poderia afetar os resultados, sendo que estes foram relatados na entrevista semi estruturada.
Não foi detectado na literatura estudos avaliando a situação dos cuidadores desses pacientes. A quase ausência de estudo publicada em revistas especializadas da área da saúde dificulta ainda mais essa orientação. Entretanto, trata-se de uma realidade a ser enfrentada pela saúde pública, que necessita a possibilidade de condições mais adequadas a esses cuidadores, estabelecendo suporte informativo sobre cuidados a serem tomados com os pacientes, adaptações a serem realizadas na casa, possibilidades de lazer, adequar meios de locomoção desses pacientes, enfim uma série de ações poderia ser proposta visando à melhora da qualidade de vida desses cuidadores e dos próprios pacientes.

Apenas um estudo ${ }^{18}$ que avaliou o grau de dependência de pessoas com perdas funcionais, ou seja, pessoas cujo grau de incapacidade para as atividades de vida diária, discriminadas como: atividades básicas da vida diária (ABVD): alimentação, vestuário, higiene, transferência e continência e, atividades instrumentais da vida diária: lavar, cozinhar, trabalhos domésticos, telefonar, utilizar meios de transporte e cuidar de medicamentos e finanças são dependentes é que constatou que $1 / 3$ destas pessoas apresentavam alto grau de dependência, ou seja, não realizavam nenhuma atividade sem ajuda. Ressalta ainda que as atividades pertinentes ao cuidador impliquem profundas mudanças no seu cotidiano, as quais freqüentemente incorrem em processos de desgaste físico e emocional que podem culminar em isolamento social, ansiedade, depressão e mesmo doenças orgânicas.

Tabela 1. Comparação entre as variávies da Escala CBE.

\begin{tabular}{|l|l|l|}
\hline Variáveis & média & valor de $\mathrm{p}$ \\
\hline Tensão geral X Isolamento & $2,33 \times 2,55$ & 0,2813 \\
\hline Tensão geral X Decepção & $2,33 \times 2,25$ & 0,3563 \\
\hline Tensão geral X Emocional & $2,33 \times 1,74$ & $0,0070^{*}$ \\
\hline Tensão geral X Ambiente & $2,33 \times 2,45$ & 0,0988 \\
\hline Isolamento X Decepção & $2,55 \times 2,25$ & 0,0750 \\
\hline Isolamento X Emocional & $2,55 \times 1,74$ & $0,0067^{*}$ \\
\hline Isolamento X Ambiente & $2,55 \times 2,45$ & 0,1270 \\
\hline Decepção X Emocional & $2,25 \times 1,74$ & 0,6685 \\
\hline Decepção X Ambiente & $2,25 \times 2,45$ & 0,1345 \\
\hline Emocional X Ambiente & $1,74 \times 2,45$ & $0,0080^{*}$ \\
\hline${ }^{*}<<0,05$ & &
\end{tabular}


Assim, equipes de saúde devem avaliar o grau de problemas de saúde existentes nos cuidadores e como estes podem interferir na tarefa de cuidar e na sua qualidade de vida.

Nota-se que transtornos de saúde em uma casa podem refletir de maneira negativa nesse ambiente, relacionado a itens de qualidade de vida. Portanto, o conceito de saúde global do individuo pode ser afetado por problemas não orgânicos, como nesse caso, pela interferência negativa de determinadas doenças em uma casa. Alerta-se para o custo econômico e social imposto por esse tipo de problema.

\section{CONCLUSÃO}

Verificaram-se neste estudo algumas variáveis que podem influenciar a QV dos cuidadores de amputados de membros inferiores. Os dados obtidos e tratados estatisticamente fornecem subsídios para esta afirmação.

Desta forma, avaliar a QV não se esgota na aplicação de um instrumento de medida, mas tem o intuito de subsidiar políticas publicas social e de saúde para esta população, sugerindo um planejamento de serviços por meio de ações que considerem as dimensões que fortalecem ou desgastem a percepção da QV destas pessoas.

\section{REFERENCIAS BIBLIOGRAFICAS}

1.Gamba MA, Gotlieb SLD, Bergamaschi DP, Vianna LAC. Amputações de extremidades inferiores por diabetes mellitus: estudo caso-controle. Rev Saúde Pub 2004;38(3):399-404

2Buzato MAS, Tribulatto EC, Costa SM, Zorn WGW. Amputados de membros inferiores: a condição dos pacientes dois anos depois. Med Circ 2001; 128(4):111-9.

3.Chamlian TR, Masiero D. Perfil epidemiológico dos pacientes amputados tratados no Centro de Reabilitaçäo Lar Escola Säo Francisco. Acta Fisatr 1998;5(1):38-42.

4.Zarit SH, Todd P, Zarit J. Subjective burden of husbands and Wives as caregivers: a longitudinal study. Gerontol 1986;2:260-6.

5.Fink SV. The Influence of family resources and family demands on the strains and well being of caregiving families. Nurs Res 1995;44(3):139-46.
6. Holmes D, Terese J, Holmes M, Bergman S, King $Y$, Bentur N. Informal vesus formal supports for impaired elderly people: determinants of choice on Israli Kibbutzin. Gerontol 1989;29:195-202.

7.Freitas MC, Santana ME. Implementaçäo da estratégia de ensino-aprendizagem à família de paciente crônico. Rev Bras Enferm 2002;55(2): 146-50. 8.Belasco AGS. Qualidade de vida dos cuidadores de pacientes com insuficiência renal crônica em hemodiálise (Tese). São Paulo: UNIFESP, 2002, 84 p. 9.Albuquerque SMLA. Qualidade de vida: diferentes concepções. In: Albuquerque SMLA. Qualidade de vida do idoso. São Paulo: Casa do Psicólogo, 2003, 53-64.

10.Amendola F. Qualidade de vida de cuidadores de familiares de pacientes com perdas funcionais e dependência atendidos pelo PSF(Dissertação). São Paulo: USP, 2007, 142p.

11.Medeiros MMC, Ferraz MB, Quaresma MR. The Caregiver Burden Scale: a Brazilian cultural adaptation and validation. Braz J Rheumatol 1998;38:1939.

12.Watanabe HAW, Dernt AM. Cuidadores de idosos: uma experiência em UBS. Rev Mun Sau 2005;29(4):639-44.

13.Juarez G, Ferrell B, Uman G, Podnos Y, Wagman LD. Distress and quality of life concerns of family caregivers of patients undergoing palliative surgery. Cancer Nurs 2008;31(1):2-10.

14.Faro ACM. Cuidar do lesado medular em casa: a vivência singular do cuidado familiar (Dissertação). São Paulo: USP, 1999, 127p.

15.Bromberg MB. Assessing quality of life in ALS. J Clin Neuromuscul Dis 2007;9(2):318-25.

16.Sorrell JM. Caring for the caregivers.J Psychosoc Nurs Ment Health Serv 2007; 45(11):17-20.

17.Simonelli C, Tripodi F, Rossi R, Fabrizi A, Lembo $D$, Cosmi V, et al. The influence of caregiver burden on sexual intimacy and marital satisfaction in couples with an Alzheimer spouse. Int J Clin Pract 2007;62(1):47-52.

18.Duayer MF, Oliveira MAC. Cuidados domiciliarios no SUS: uma resposta as necessidades especiais de pessoas com perdas funcionais e dependência. Rev Saúde Deb 2003;12:23-8. 